\title{
The New Identity for the Scattering Matrix of Exactly Solvable Models
}

\author{
Vladimir Korepin ${ }^{\dagger}$ and Nikita Slavnov ${ }^{\ddagger}$ \\ † ITP, SUNY at Stony Brook, NY 11794-3840, USA. \\ korepin@insti.physics.sunysb.edu \\ $\ddagger$ Steklov Mathematical Institute, Gubkina 8, Moscow 117966, Russia. \\ nslavnov@mi.ras.ru
}

\begin{abstract}
We discovered a simple quadratic equation, which relates scattering phases of particles on Fermi surface. We consider one dimensional Bose gas and $X X Z$ Heisenberg spin chain.
\end{abstract}

\footnotetext{
${ }^{1}$ Dedicated to J. Zittartz on the occasion of his 60 th birthday
} 


\section{Introduction}

To define everything precisely we consider a specific model. Let us concentrate our attention on Bose gas with delta interaction (quantum Nonlinear Schrödinger equation).

The Hamiltonian of the model is

$$
H=\int d x\left(\partial_{x} \Psi^{\dagger}(x) \partial_{x} \Psi(x)+c \Psi^{\dagger}(x) \Psi^{\dagger}(x) \Psi(x) \Psi(x)-h \Psi^{\dagger}(x) \Psi(x)\right) .
$$

Here $0<c<\infty, h>0$ are the coupling constant and the chemical potential respectively. The canonical Bose fields $\Psi(x, t), \Psi^{\dagger}(x, t),(x, t \in \mathbf{R})$ obey the standard commutation relations

$$
\left[\Psi(x, t), \Psi^{\dagger}(y, t)\right]=\delta(x-y)
$$

and act in the Fock space with the vacuum vector $|0\rangle$, which is characterized by the relation:

$$
\Psi(x, t)|0\rangle=0 \text {. }
$$

Alternatively the model can be formulated on the language of many-body quantum mechanics system, containing $N$ identical particles. In this case the Hamiltonian of the Bose gas can be represented as

$$
\mathcal{H}_{N}=-\sum_{j=1}^{N} \frac{\partial^{2}}{\partial x_{j}^{2}}+2 c \sum_{N \geq j>k \geq 1} \delta\left(x_{j}-x_{k}\right)-h N .
$$

For nonzero value of the coupling constant the Pauli principal is valid (chapter VII of [1]).

The model was solved by Bethe Ansatz [2]. The ground state is a Fermi sphere. In order to describe it precisely it is convenient to introduce spectral parameter $\lambda$ (similar to rapidity). The derivative of the momentum of the particle with respect to the spectral parameter is

$$
\frac{\partial k(\lambda)}{\partial \lambda}=2 \pi \rho(\lambda)
$$

where the function $\rho(\lambda)$ is defined by an integral equation

$$
\rho(\lambda)-\frac{1}{2 \pi} \int_{-q}^{q} K(\lambda, \mu) \rho(\mu) d \mu=\frac{1}{2 \pi} .
$$


Here $q$ is the value of the spectral parameter on the Fermi surface, and

$$
K(\lambda, \mu)=\frac{2 c}{c^{2}+(\lambda-\mu)^{2}} .
$$

One can prove that the integral operator $\hat{I}-\frac{1}{2 \pi} \hat{K}$ is not degenerated, and hence, the equation (1.6) has unique solution ([3], chapter I of [1]). The density of the gas is given by

$$
D=\int_{-q}^{q} \rho(\lambda) d \lambda
$$

There is one particle in the model. It is defined at $\lambda \geq q$ or $\lambda \leq-q$. The energy of the particle $\varepsilon(\lambda)$ is

$$
\varepsilon(\lambda)-\frac{1}{2 \pi} \int_{-q}^{q} K(\lambda, \mu) \varepsilon(\mu) d \mu=\lambda^{2}-h .
$$

It vanishes on the Fermi surface $\varepsilon( \pm q)=0$. The momentum is

$$
k(\lambda)=\lambda+\int_{-q}^{q} \theta(\lambda-\mu) \rho(\mu) d \mu .
$$

Here

$$
\theta(\lambda)=i \ln \left(\frac{i c+\lambda}{i c-\lambda}\right)
$$

One can calculate a scattering matrix of particle with spectral parameter $\lambda$ on another particle with spectral parameter $\mu$ (chapter I of [1]). There is no multi-particle production or reflection. Transition coefficient is

$$
\exp \{2 \pi i F(\lambda \mid \mu)\}
$$

The phase $F(\lambda \mid \mu)$ is defined by an integral equation

$$
F(\lambda \mid \mu)-\frac{1}{2 \pi} \int_{-q}^{q} K(\lambda, \nu) F(\nu \mid \mu) d \nu=\frac{1}{2 \pi} \theta(\lambda-\mu) .
$$

The most important are scattering phases of particles on the Fermi edges $F(q \mid q)$ and $F(q \mid-q)$. In this paper we prove the identity

$$
\operatorname{det}\left(\begin{array}{cc}
1-F(q \mid q) & F(q \mid-q) \\
-F(-q \mid q) & 1+F(-q \mid-q)
\end{array}\right)=1 .
$$


This is the main result of the paper. Another way to rewrite this identity is

$$
(1-F(q \mid q))^{2}-F^{2}(q \mid-q)=1 \text {. }
$$

Here we have used the property $F(-\lambda \mid-\mu)=-F(\lambda \mid \mu)$, which follows immediately from the antisymmetry of $\theta(\lambda-\mu)=-\theta(\mu-\lambda)$.

This identity also permit us to relate "fractional" charge to the phase shift on the Fermi surface. Fractional charge $\mathcal{Z}$ appears in formulæ for finite size corrections (chapter I of [1]). This value is necessary for conformal description of the model (chapter XVIII of [1]) and it is equal to

$$
\mathcal{Z}=2 \pi \rho(q) \text {. }
$$

Using the equations (1.6), (1.13) for $\rho(\lambda)$ and $F(\lambda \mid \mu)$, one can find the relationship between the fractional charge and the scattering phase on the Fermi surface

$$
\mathcal{Z}=1+F(q \mid-q)-F(q \mid q)
$$

Indeed, it follows from (1.6) that

$$
[2 \pi \rho(\lambda)-1]-\frac{1}{2 \pi} \int_{-q}^{q} K(\lambda, \mu)[2 \pi \rho(\mu)-1] d \mu=\frac{1}{2 \pi}[\theta(\lambda+q)-\theta(\lambda-q)] .
$$

Comparing this equation with (1.13) we find

$$
2 \pi \rho(\lambda)=1+F(\lambda \mid-q)-F(\lambda \mid q),
$$

what, in turns, implies (1.17). The identity (1.15) allows us to find new relation

$$
\mathcal{Z}^{-1}=1-F(q \mid-q)-F(q \mid q) .
$$

\section{The proof of the main identity}

In this section we give the proof of the identity (1.14). In order to do this, one should calculate the derivatives of the function $F(\lambda \mid \mu)$ with respect to $\lambda, \mu$ and $q$. Using the basic equation (1.13), we have

$$
\begin{array}{rl}
\frac{\partial F(\lambda \mid \mu)}{\partial \lambda}-\frac{1}{2 \pi} \int_{-q}^{q} & K(\lambda, \nu) \frac{\partial F(\nu \mid \mu)}{\partial \nu} d \nu=\frac{1}{2 \pi} K(\lambda, \mu) \\
& -\frac{1}{2 \pi} K(\lambda, q) F(q \mid \mu)+\frac{1}{2 \pi} K(\lambda,-q) F(-q \mid \mu),
\end{array}
$$




$$
\begin{gathered}
\frac{\partial F(\lambda \mid \mu)}{\partial \mu}-\frac{1}{2 \pi} \int_{-q}^{q} K(\lambda, \nu) \frac{\partial F(\nu \mid \mu)}{\partial \mu} d \nu=-\frac{1}{2 \pi} K(\lambda, \mu), \\
\frac{\partial F(\lambda \mid \mu)}{\partial q}-\frac{1}{2 \pi} \int_{-q}^{q} K(\lambda, \nu) \frac{\partial F(\nu \mid \mu)}{\partial q} d \nu \\
=\frac{1}{2 \pi} K(\lambda, q) F(q \mid \mu)+\frac{1}{2 \pi} K(\lambda,-q) F(-q \mid \mu) .
\end{gathered}
$$

Here we have used that $\frac{\partial}{\partial \lambda} \theta(\lambda-\mu)=K(\lambda, \mu)$.

As we have mentioned already, the resolvent of the operator $\hat{I}-\frac{1}{2 \pi} \hat{K}$ exists and it is equal to

$$
\hat{R}=\left(\hat{I}-\frac{1}{2 \pi} \hat{K}\right)^{-1} \cdot \frac{1}{2 \pi} \hat{K}
$$

The derivatives of the function $F(\lambda \mid \mu)$ can be expressed in terms of the resolvent

$$
\begin{aligned}
& \frac{\partial F(\lambda \mid \mu)}{\partial \lambda}=R(\lambda, \mu)-R(\lambda, q) F(q \mid \mu)+R(\lambda,-q) F(-q \mid \mu) \\
& \frac{\partial F(\lambda \mid \mu)}{\partial \mu}=-R(\lambda, \mu) \\
& \frac{\partial F(\lambda \mid \mu)}{\partial q}=R(\lambda, q) F(q \mid \mu)+R(\lambda,-q) F(-q \mid \mu)
\end{aligned}
$$

Using these equations one can find the complete derivatives with respect to $q$ of functions $F(q \mid q), F(q \mid-q)$ etc.:

$$
\begin{aligned}
& \frac{d}{d q} F(q \mid \pm q)=\left.\left(\frac{\partial}{\partial \lambda} \pm \frac{\partial}{\partial \mu}+\frac{\partial}{\partial q}\right) F(\lambda \mid \mu)\right|_{\substack{\lambda=q \\
\mu= \pm q}}, \\
& \frac{d}{d q} F(-q \mid \pm q)=\left.\left(-\frac{\partial}{\partial \lambda} \pm \frac{\partial}{\partial \mu}+\frac{\partial}{\partial q}\right) F(\lambda \mid \mu)\right|_{\substack{\lambda=-q \\
\mu= \pm q}} .
\end{aligned}
$$


Substituting here equations (2.5) we find

$$
\begin{aligned}
& \frac{d}{d q} F(q \mid-q)=2 R(q,-q)(1+F(-q \mid-q)), \\
& \frac{d}{d q} F(-q \mid q)=-2 R(-q, q)(1-F(q \mid q)), \\
& \frac{d}{d q} F(q \mid q)=2 R(q,-q) F(-q \mid q), \\
& \frac{d}{d q} F(-q \mid-q)=2 R(-q, q) F(q \mid-q) .
\end{aligned}
$$

Now it is sufficient to take the derivative with respect to $q$ of the l.h.s. of the equation (11.14):

$$
\begin{gathered}
\frac{d}{d q} \operatorname{det}\left(\begin{array}{cc}
1-F(q \mid q) & F(q \mid-q) \\
-F(-q \mid q) & 1+F(-q \mid-q)
\end{array}\right) \\
=-\frac{d F(q \mid q)}{d q}(1+F(-q \mid-q))+(1-F(q \mid q)) \frac{d F(-q \mid-q)}{d q} \\
\quad+\frac{d F(q \mid-q)}{d q} F(-q \mid q)+F(q \mid-q) \frac{d F(-q \mid q)}{d q} \\
=-2 R(q,-q) F(-q \mid q)(1+F(-q \mid-q)) \\
+2 R(-q, q) F(q \mid-q)(1-F(q \mid q)) \\
+2 R(q,-q) F(-q \mid q)(1+F(-q \mid-q)) \\
-2 R(-q, q) F(q \mid-q)(1-F(q \mid q))=0
\end{gathered}
$$


On the other hand, it is clear that for $q=0$ we have

$$
\left.\operatorname{det}\left(\begin{array}{cc}
1-F(q \mid q) & F(q \mid-q) \\
-F(-q \mid q) & 1+F(-q \mid-q)
\end{array}\right)\right|_{q=0}=1
$$

Thus, the identity (1.14) is proved.

We would like to emphasize the we did not use the explicit expressions for the kernel $K(\lambda, \mu)$ and the function $\theta(\lambda-\mu)$. In fact, we have used only three properties:

a) the existence of the resolvent of the operator $\hat{I}-\frac{1}{2 \pi} \hat{K}$;

b) the kernel $K(\lambda, \mu)$ and the function $\theta(\lambda-\mu)$ depend on the difference;

c) the derivative of $\theta(\lambda-\mu)$ is equal to the kernel $K(\lambda, \mu)$.

In order to reduce (1.14) to the identity (1.15) one should use also the antisymmetry property $\theta(\lambda-\mu)=-\theta(\mu-\lambda)$.

Thus, the quadratic identity for the scattering phase is valid for a wide class of completely integrable models, but not only for the one-dimensional Bose gas. In particular, it is valid for scattering phases of elementary particles (spin waves) of $X X Z$ Heisenberg spin chain in a magnetic field (chapter II of [1]).

\section{Acknowledgments}

This work was supported by the National Science Foundation (NSF) Grant No. PHY-9321165, and the Russian Foundation of Basic Research Grant No. 96-01-00344.

\section{References}

[1] V. E. Korepin, N. M. Bogoliubov and A. G. Izergin, Quantum Inverse Scattering Method and Correlation Functions (Cambridge University Press, 1993)

[2] E. H. Lieb and W. Liniger, Phys. Rev. 130,1605 (1963)

[3] C. N. Yang and C. P. Yang, J. Math. Phys. 10,1115 (1969) 